\title{
Competitive Interactions Between Incompatible Mutants of the Social Bacterium Myxococcus xanthus DK1622
}

\section{OPEN ACCESS}

Edited by:

Alexandre Jousset,

Utrecht University, Netherlands

Reviewed by:

Xiuzhu Dong,

Institute of Microbiology (CAS), China

Cyrus Alexander Mallon,

University of Groningen, Netherlands

${ }^{*}$ Correspondence:

Yue-zhong L

lilab@sdu.edu.cn

Specialty section:

This article was submitted to

Terrestrial Microbiology,

a section of the journal

Frontiers in Microbiology

Received: 26 June 2017

Accepted: 16 May 2018

Published: 05 June 2018

Citation:

Gong Y, Zhang Z, Zhou X, Anwar MN, Hu X, Li Z, Chen X and Li Y (2018) Competitive Interactions Between Incompatible Mutants of the Social Bacterium Myxococcus xanthus DK1622. Front. Microbiol. 9:1200 doi: 10.3389/fmicb.2018.01200

\section{Ya Gong, Zheng Zhang, Xiu-wen Zhou, Mian N. Anwar, Xiao-zhuang Hu, Ze-shuo Li, Xiao-jing Chen and Yue-zhong $\mathrm{Li}^{*}$}

State Key Laboratory of Microbial Technology, School of Life Science, Shandong University, Jinan, China

Due to the high similarity in their requirements for space and food, close bacterial relatives may be each other's strongest competitors. Close bacterial relatives often form visible boundaries to separate their swarming colonies, a phenomenon termed colony-merger incompatibility. While bacterial species are known to have many incompatible strains, it is largely unclear which traits lead to multiple incompatibilities and the interactions between multiple incompatible siblings. To investigate the competitive interactions of closely related incompatible strains, we mutated Myxococcus xanthus DK1622, a predatory bacterium with complex social behavior. From 3392 random transposon mutations, we obtained 11 self-identification (SI) deficient mutants that formed unmerged colony boundaries with the ancestral strain. The mutations were at nine loci with unknown functions and formed nine independent SI mutants. Compared with their ancestral strain, most of the SI mutants showed reduced growth, swarming and development abilities, but some remained unchanged from their monocultures. When pairwise mixed with their ancestral strain for co-cultivation, these mutants exhibited improved, reduced or unchanged competitive abilities compared with the ancestral strain. The sporulation efficiencies were affected by the DK1622 partner, ranging from almost complete inhibition to 360\% stimulation. The differences in competitive growth between the SI mutants and DK1622 were highly correlated with the differences in their sporulation efficiencies. However, the competitive efficiencies of the mutants in mixture were inconsistent with their growth or sporulation abilities in monocultures. We propose that the colony-merger incompatibility in $M$. xanthus is associated with multiple independent genetic loci, and the incompatible strains hold competitive interaction abilities, which probably determine the complex relationships between multiple incompatible $M$. xanthus strains and their co-existence strategies.

Keywords: Myxococcus xanthus, transposon-mutation, multiple genetic loci, self-identification, competitive interaction, colony-merger incompatibility 


\section{INTRODUCTION}

Hamilton's kin selection rule asserts that the closer the kin relationship between individuals, the stronger their cooperative tendencies and altruistic behavior (Hamilton, 1964; West et al., 2006; Strassmann et al., 2011; Waibel et al., 2011). However, because of their highly similar requirements for space and food, close relatives are potentially the strongest competitive neighbors in nature. Kin discrimination provides a means for individuals to identify self and non-self, thus separating competitive neighbors and probably aiding the survival of different kin groups. Closely related strains represent the basic units of bacterial communities in nature (Hamilton, 1964; West et al., 2006; Strassmann et al., 2011; Waibel et al., 2011). Many bacterial cells are able to discriminate self from non-self relatives by forming boundaries between encountered colonies (Dienes, 1946; Munson et al., 2002; Gibbs et al., 2008; Vos and Velicer, 2009; Rendueles et al., 2015; Stefanic et al., 2015; Lyons et al., 2016). Colony-merger incompatibility is a type of bacterial kin discrimination that results in the separation of different groups, and probably allows the existence of diverse incompatible strains within bacterial species in a small patch of soil. For example, a centimeter-scale soil sample yielded 45 incompatible types when 78 Myxococcus xanthus isolates were pairwise inoculated in close proximity on growth medium (Vos and Velicer, 2009). Similarly, among 39 isolates of Bacillus subtilis from two $1-\mathrm{cm}^{3}$ soil samples, 12 incompatible types were identified with striking boundaries between their swarms (Stefanic et al., 2015). The incompatible characteristics and the interactions between incompatible siblings are suggested to be important for understanding the co-existence mechanisms of incompatible groups and their ecological functions (Griffin et al., 2004; Keller and Surette, 2006; West et al., 2007; Brown and Buckling, 2008; Velicer and Vos, 2009; Li et al., 2013). Gibbs and Greenberg proposed that the boundaries formed between incompatible bacterial strains can establish and harbor an area in which one strain occupies the space and food exclusively and protects itself against colonization by a competing strain (Gibbs and Greenberg, 2011). Such a colony boundary is also suggested to be a barrier to homologous recombination (gene flow) between closely related incompatible strains, which may promote ecological divergence and co-existence (Wielgoss et al., 2016). Natural incompatible sibling isolates normally have high mutation frequencies and horizontal gene transfers, which make the comparative genomics of natural isolates difficult to interpret with regard to the incompatibility of phenotypes. Incompatible laboratory mutants derived from the same ancestral strains can contribute to our understanding of the incompatibility characteristics and the interactions between incompatible siblings, and also facilitate further research.

Myxococcus xanthus displays complex social behavior (Shimkets, 1990; Dworkin, 1996). M. xanthus cells locomote on solid surfaces in swarms and prey collaboratively on other microbial cells in a wolf-pack pattern. When food is scarce, the $M$. xanthus cells aggregate to develop multicellular fruiting bodies, inside which stress-resistant myxospores are formed. Social behavior occurs between the cells of a single Myxococcus strain. If two different Myxococcus species or strains of the same species are co-cultured, they might occupy separate territories (Smith and Dworkin, 1994; Vos and Velicer, 2009). The number of incompatible strains-for example, 45 incompatible types in the $78 \mathrm{M}$. xanthus centimeter-scale isolates (Vos and Velicer, 2009)-suggests that closely related incompatible siblings may possess various features and their interactions are entirely competitive. However, these previous studies were performed with Myxococcus natural strains with unclear genetic backgrounds, leaving the underlying mechanisms unclear. In this study, to investigate the traits leading to the multiple incompatibilities and the competitive interactions between multiple incompatible siblings, we mutated M. xanthus DK1622, a model myxobacterium strain, by random transposon insertion mutagenesis and obtained multiple colony-merger incompatible mutants. We checked the cellular growth abilities and social characteristics of these self-identification (SI) mutants in monocultures to determine their relevance to the colony-merger incompatibility. We assayed their competitive interactions in co-cultures during growth and sporulation and found that the mutants exhibited improved, reduced or unchanged competitive abilities in mixture; however, these abilities were not relevant to their growth or development abilities in monoculture. We determined that multiple colony-merger incompatibilities in $M$. xanthus are associated with different genetic loci and interactions between the incompatible strains are diverse, reflecting the diversity of the underlying mechanisms of incompatibility. We discuss the significance of competitive interactions between co-existing incompatible close relatives in nature.

\section{RESULTS}

\section{Colony-Merger Incompatibility Mutations in Myxococcus xanthus DK1622}

We made insertion mutations in $M$. xanthus DK1622 using the pMiniHimar-lacZ plasmid, which is able to insert into genomes randomly (Rubin et al., 1999; Youderian et al., 2003). To screen for incompatible mutants that formed visible colony boundaries with neighbors, transformants were inoculated adjacent to each other on CTT growth medium (Hodgkin and Kaiser, 1977) supplemented with kanamycin. Figure 1A shows an incompatible mutant with its neighboring mutants. The incompatible kanamycin-resistant recombinants were further inoculated adjacent to the wild-type strain on a CTT plate to confirm their colony-merger incompatibilities. From 3,392 mutations, we obtained 11 mutants that formed visible colony boundaries with DK1622, while two colonies of DK1622 or the same mutant strain were merged (Figure 1B). Southern blotting experiments indicated that each of the 11 mutants contained a single insertion in the genome. We named these incompatible mutants as SI mutants SI01-SI11. We examined the boundary formation between the incompatible strains under a microscope. In two adjacently inoculated incompatible colonies, the cells that moved toward the opposite colonies stopped moving forward when encountered. The accumulated cells 


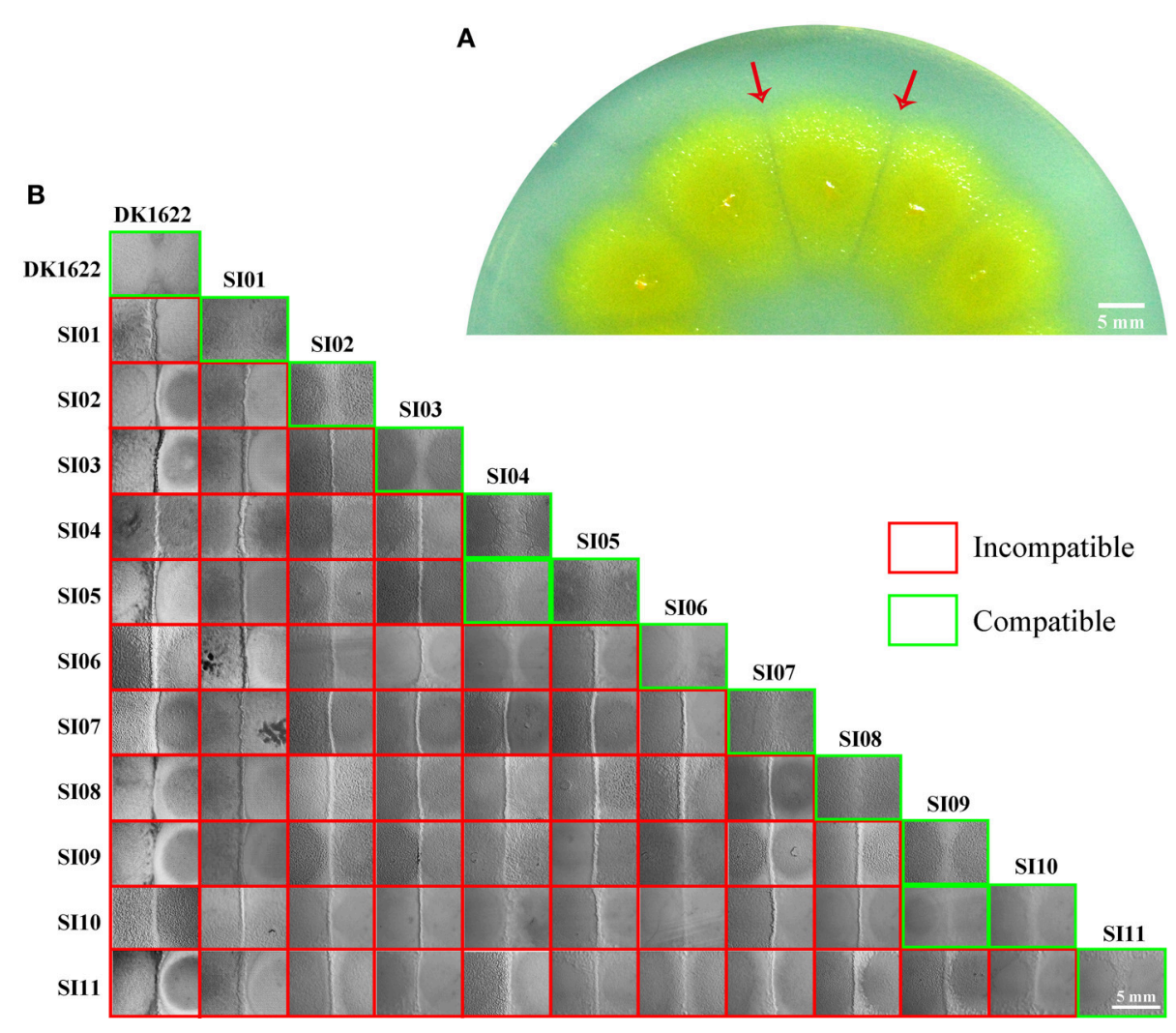

FIGURE 1 | Screening for incompatible mutants in M. xanthus DK1622. (A) Visible boundaries (red arrows) formed between two adjacent colonies of pMiniHimar-lacZ insertion mutants on CTT agar plates supplemented with kanamycin. (B) The colony development between the 11 SI mutants and DK1622. All photos are representative of three biological replicates. Scale bar, $5 \mathrm{~mm}$ (A,B).

protruded vertically at the colony edges, thus forming a boundary ravine separating the two colonies. Video $\mathbf{S 1}$ demonstrates the boundary formation between the SI01 mutant and the wildtype DK1622. Furthermore, a dyeing experiment showed that the colony boundaries between SI mutants and the wild-type strain might contain damaged cells (Figure $\mathbf{S 1}$ demonstrates the dyeing result of the adjacent colonies of DK1622 and SI04).

Using plasmid-rescuing techniques, we located the insertion sites of the SI mutants. Sequencing revealed that the 11 mutations were at 9 genetic loci scattered throughout the genome of $M$. xanthus DK1622 (NC_008095.1; Table 1). All of the 11 mutations were located in open reading frames, including three newly annotated genes (MXAN_RS36575 in SI05, MXAN_RS24590 in SI08 and MXAN_RS34540 in SI10). Three of these genes were annotated to encode a putative regulator of ribonuclease A (MXAN_0390, SI03; Goldman et al., 2006), a putative methyltransferase (MXAN_1599, SI06) and a serine/threonine protein kinase (MXAN_7251, SI11), while the others were hypothetical open reading frames. These mutated genes have not yet been studied genetically or biochemically. We further tried to delete each of the insertion-mutated genes in the wild-type strain DK1622, and six deletion mutants were obtained and exhibited similar incompatibilities to the insertion mutants; i.e., the deletion mutants merged their colonies with the corresponding insertion mutants, but formed colony boundaries with the wild-type strain (Figure S2). The six genes were MXAN_0049 in SI01, MXAN_0085 in SI02, MXAN_RS36575 in SI05, MXAN_2099 in SI07, MXAN_RS24590 in SI08 and MXAN_RS34540 in SI10, suggesting that these six genes were responsible for the colonymerger incompatibility.

When these mutants were pairwise inoculated adjacent to each other on a CTT plate, two pairs (SI04/SI05 and SI09/SI10) formed merged colonies, while the other pairs were separated from each other by an obvious colony boundary (Figure 1B). Notably, the two pairs that merged colonies had close insertion sites, consistent with their similarly compatible phenotypes. The insertion site in the SI04 mutant was in MXAN_1307, while the mutation in SI05 was in a new gene upstream to MXAN_1307. Similarly, the mutation in the SI09 mutant was located in MXAN_7134, while the SI10 mutant was inserted in a newly annotated gene upstream to MXAN_7134 (Table 1). Thus, the 11 SI mutants formed 9 independent incompatible mutants that were derived from 9 different genetic determinants. Because the nine insertion-mutated genetic loci were scattered throughout the genome of $M$. xanthus (Table 1), we propose that the nine independent genetic determinants might be involved in colonymerger incompatibility. 
TABLE 1 | Eleven SI mutants and their information.

\begin{tabular}{|c|c|c|c|}
\hline Mutants & Locus inserted & $\begin{array}{l}\text { Length } \\
\text { (bp) }\end{array}$ & $\begin{array}{l}\text { Putative } \\
\text { identification }\end{array}$ \\
\hline SI01 & MXAN_0049 & 585 & hypothetical protein \\
\hline $\mathrm{SIO2}$ & MXAN_0085 & 588 & hypothetical protein \\
\hline $\mathrm{SIO3}$ & MXAN_0390 & 498 & $\begin{array}{l}\text { regulator of } \\
\text { ribonuclease activity A, } \\
\text { putative }\end{array}$ \\
\hline $\mathrm{SIO} 4$ & MXAN_1307 & 867 & hypothetical protein \\
\hline SI05 & $\begin{array}{l}\text { MXAN_RS36575 (between } \\
\text { MXAN_1307 and MXAN_1308) }\end{array}$ & 585 & hypothetical protein \\
\hline SI06 & MXAN_1599 & 1,500 & $\begin{array}{l}\text { putative } \\
\text { methyltransferase }\end{array}$ \\
\hline SIO7 & MXAN_2099 & 561 & $\begin{array}{l}\text { conserved hypothetical } \\
\text { protein }\end{array}$ \\
\hline SI08 & $\begin{array}{l}\text { MXAN_RS24590 (between } \\
\text { MXAN_5062 and MXAN_5063) }\end{array}$ & 462 & hypothetical protein \\
\hline SI09 & MXAN_7134 & 777 & hypothetical protein \\
\hline Sl10 & $\begin{array}{l}\text { MXAN_RS34540 (between } \\
\text { MXAN_7134 and MXAN_7135) }\end{array}$ & 588 & hypothetical protein \\
\hline Sl11 & MXAN_7251 & 3,627 & $\begin{array}{l}\text { serine/threonine protein } \\
\text { kinase }\end{array}$ \\
\hline
\end{tabular}

\section{The SI Mutants Showed Variable Growth Abilities and Retained Multicellular Social Behavior}

Cell-to-cell self-recognition is a prerequisite for Myxococcus strains to perform multicellular social behavior, such as the formation of fruiting bodies and social motility (S-motility). The nine independent SI mutants from DK1622 formed incompatible colonies with their parent strain, as well as with each other, but these SI mutants each merged colonies by themselves. In monocultures, the SI mutants had variable growth abilities (Figure 2A). Compared with the wild-type strain, some mutants, such as SI01, SI03, SI04, and SI11, produced more than 10 times fewer colony-forming units (CFUs) after 48-h incubation. In contrast, the CFUs of some mutants, such as SI09, were similar to those of the wild-type strain.

Incompatibility occurs between two swarming colonies. The cellular motility of these SI mutants should play a role in the formation of colony-merger incompatibility. Both type IV pili (TFP) and extracellular polysaccharides (EPS) are essential to the social behavior of M. xanthus cells (Wu and Kaiser, 1997; Li et al., 2003). We performed Western blot experiments with the antibodies of PilA proteins (the major component of TFP) and demonstrated that the SI mutants maintained the pilus formation ability (Figure S3A). Quantitative dye binding with Congo red and qualitative colony staining with calcofluor white experiments (Black and Yang, 2004) showed that the mutants had the same ability to produce EPS as the wild-type strain (Figures S3B,C).

We assayed the swarm sizes of the SI mutants on soft $(0.3 \%$ agar) and hard (1.5\% agar) CTT medium to determine the motility of Myxococcus cells (Shi and Zusman, 1993). The colony diameters ranged from 83 to $106 \%$ of DK1622 on the soft agar plate and from 78 to $104 \%$ of DK1622 on the hard agar plate (Figure 2B), which suggested that the mutants retained a similar swarming ability to the wild-type strain. We checked the sporulation abilities of the SI mutants on TPM medium, which contains no nutrients and is normally used for assays of the development ability of Myxococcus cells (Kroos et al., 1986). All of the SI mutants were able to form multicellular fruiting bodies (Figure 2C). The sporulation abilities of these SI mutants varied from 40 to $86 \%$ of the wild-type strain (Figure 2D). The results suggest that compared with their ancestral strain, most of the SI mutants had reduced growth, swarming and development abilities, and some remained unchanged in the monoculture.

\section{Sporulation Mixing Efficiency of the Nine SI Mutants and DK1622}

To investigate the interactions between incompatible strains, we mixed the wild-type strain with each of the nine independent SI mutants and cultivated the mixtures on the TPM development medium. The mixtures of these pairs all formed multicellular fruiting bodies, with similar morphologies to those in their monocultures (Figure 3A). After 5 days of incubation, we checked the sporulation abilities of the partners in each pair mixture based on the kanamycin-resistance characteristics of the SI mutants and the sensitiveness of DK1622. Interestingly, the sporulation abilities of the SI mutants, as well as the wildtype strain, varied in different paired mixtures (Figure S4A). We used the $W_{S I-D K}$ parameter to quantify the sporulation mixing efficiency of a given SI mutant $(S I)$ and DK1622 (DK) in the co-development experiments (Figure S4B). While the $W_{S I-D K}$ values were not significantly changed for SI03 ( $t$-test, $p=0.36$ ) and SI11 ( $t$-test, $p=0.11$ ), those for the other seven SI mutants and DK1622 pairs were negative ( $t$-test, $p<0.01$ ). The negative values of $W_{S I-D K}$ indicated that the sporulation efficiency of the SI mutant partner was lower than that of DK1622 in the mixture.

Because the sporulation abilities of the mutants varied in their monocultures, we further used the $C_{S I}(D K)$ or $C_{D K}(S I)$ parameter to deduct the effects of the strain's sporulation difference from the measurement of the partners' sporulation efficiency in the mixture (Figure 3B). A positive or negative $C_{S I}(D K)$ value indicates that the SI mutant sporulates more or less efficiently in the presence of DK1622 than in monoculture; it is similar to the $C_{D K}(S I)$ parameter. Compared with that in the monoculture, the sporulation of SI01, SI04, and SI07 was significantly reduced in the mixture with DK1622 ( $t$-test, $p=0.02$ for SI04; $p<0.01$ for SI01 and SI07). For example, SI07 strongly decreased its sporulation efficiency when mixed for co-development with DK1622, and produced $\sim 17$ times fewer spores than in the monoculture $\left(C_{S I 07}[D K]=-1.22\right)$. In contrast, the sporulation efficiency of SI09 was not significantly changed ( $t$-test, $p=0.13$ ), while the other five SI mutants sporulated more efficiently in the mixtures than in the monocultures ( $t$-test, $p=0.01$ for SI06; $p<0.01$ for other SI mutants). Notably, except for the DK1622 and SI01 pair ( $t$-test, $p=0.11)$, the DK1622 strain showed significantly greater sporulation efficiency in the co-development with each of the other SI mutants than in the monoculture ( $t$-test, $p=0.01$ for the 
A

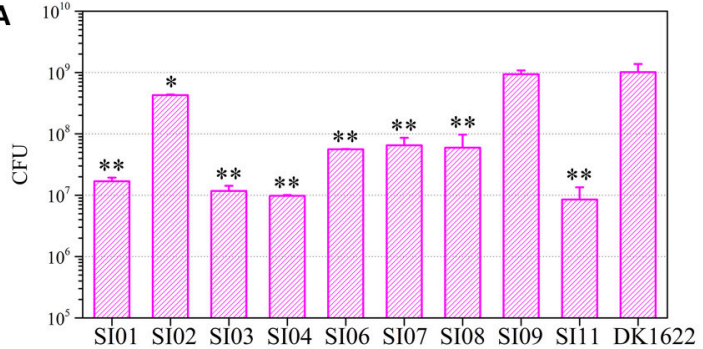

D

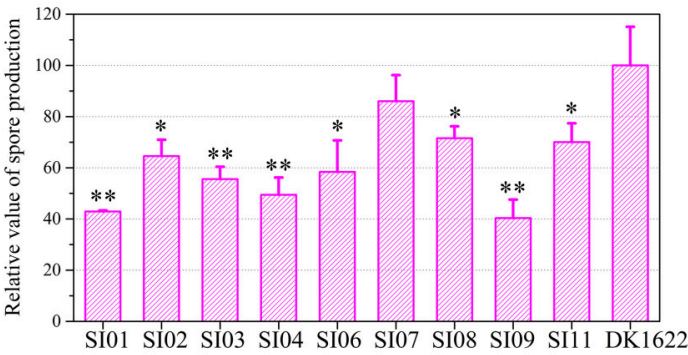

B

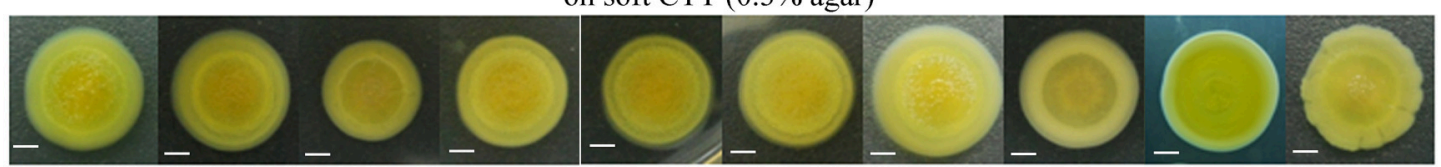

on hard CTT (1.5\% agar)

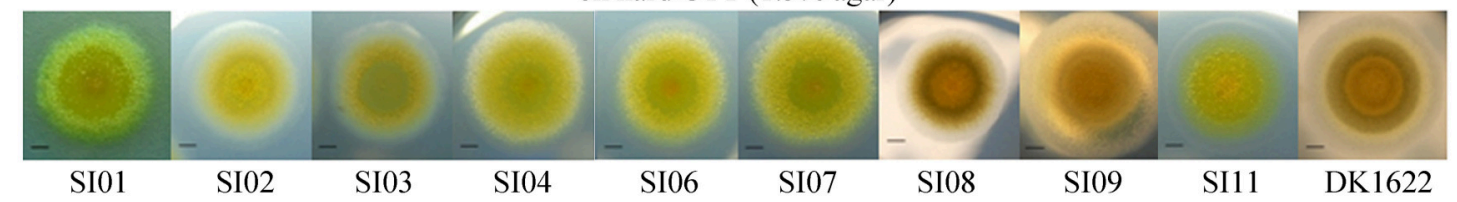

C

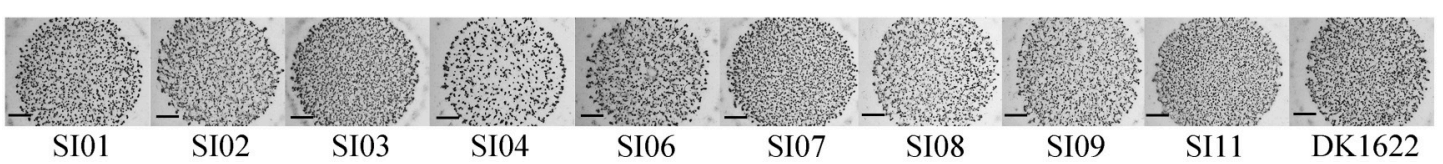

FIGURE 2 | Characteristics of incompatible mutants. (A) Colony-forming unit (CFU) counts of incompatible mutants and DK1622 after incubation for 48 hr on CTT medium. (B) Colony expansions on CTT plates containing $0.3 \%$ agar and 1.5\% agar, respectively. The pictures were taken after 5 days of incubation. (C) The developmental aggregation of the strains on TPM medium. The pictures were taken after $72 \mathrm{~h}$ of incubation. The bottom of the figure shows the strains used for fruiting body formation. The scale bars in (B,C) represent $1 \mathrm{~mm}$. (D) The relative sporulation production of the strains on TPM medium. Error bars represent the standard deviation from three independent experiments.
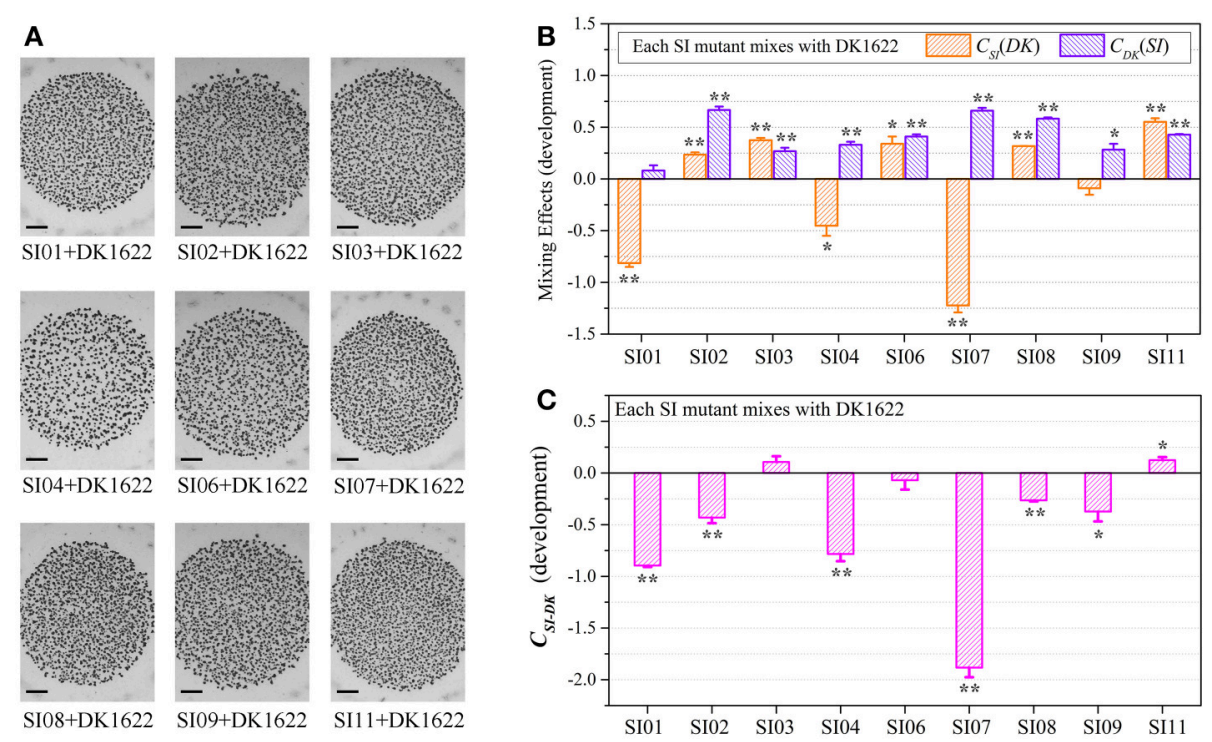

FIGURE 3 | Co-development of incompatible mutants and their ancestral strain DK1622 on the TPM medium. (A) Morphologies of the fruiting bodies of the co-cultures. The scale bars represent $1 \mathrm{~mm}$. (B) The log-scale difference between each strain's sporulation ability in mixture and in monoculture. (C) The competitive sporulation differences of co-cultured partners after eliminating the strain's sporulation difference in monoculture. Three dilutions and three replications were performed for each experiment. The error bars represent the standard deviation, and asterisks denote $p$-values for $t$-tests of differences from zero: ${ }^{\star} p<0.05$, ${ }^{* *} p<0.01$. 
pair with SI09; $p<0.01$ for the others). For example, in the codevelopment pair of DK1622 and SI07, the sporulation efficiency of DK1622 increased and produced $\sim 5$ times more spores than in the monoculture $\left(C_{D K}[S I 07]=0.66\right)$.

The $C_{S I-D K}$ parameter represents the sporulation difference between an SI mutant and DK1622 in the mixture after eliminating their sporulation difference in the monoculture (Figure 3C). We found that the $\mathrm{C}_{S I-D K}$ and $W_{S I-D K}$ parameters were highly correlated $(r=0.98, p<0.01)$. In the co-developed pairs of SI mutants and DK1622, most mutants exhibited significantly weaker competitive sporulation efficiencies than that of DK1622 ( $t$-test, $p=0.02$ for SI09; $p<0.01$ for other SI mutants), while SI11 showed a slight competitive advantage over DK1622 in the mixture $\left(\mathrm{C}_{S I 11-D K}=0.12\right.$; $t$-test, $\left.p=0.02\right)$. The sporulation efficiencies of SI03 and SI06 were not significantly affected by DK1622 in the mixtures ( $t$-test, $p=0.08$ for SI03; $p=0.31$ for SI06).

\section{Competitive Growth Abilities Between Incompatible Strains}

We assessed the survival cells of the partner strains in the paired mixtures of SI mutants and DK1622 based on their resistance and sensitivity to the kanamycin antibiotic. The SI mutants and the wild-type strain were mixed $(1: 1, \mathrm{v} / \mathrm{v})$ and incubated on a CTT plate for $48 \mathrm{~h}$. In the paired mixture, the SI mutants were all outnumbered by the wild-type strain, but to different extents (Figure S5A). In the SI01-DK1622 and SI07-DK1622 mixtures, the numbers of DK1622 cells were $(2.49 \pm 0.32) \times$ $10^{8}$ and $(1.25 \pm 0.35) \times 10^{8}$, similar to the number in its monoculture, whereas the numbers of SI01 and SI07 mutants were reduced to $(9.67 \pm 5.69) \times 10^{2}$ and $(1.50 \pm 0.70) \times 10^{2}$ after $48 \mathrm{~h}$ of incubation on the CTT growth medium, respectively. The growth abilities of the other seven independent SI mutants were weakly increased or decreased, compared with those of their monocultures. For example, while the mutants weakly increased their growth abilities in the SI03-DK1622 mixture $(t$ test, $p=0.013)$ and SI11-DK1622 mixture ( $t$-test, $p<0.01$ ), the cell numbers of the mutant strains in the SI02-DK1622, SI06-DK1622 and SI09-DK1622 mixtures decreased.

We calculated the $W_{S I-D K}$ parameter to estimate the competitive abilities between the SI mutants (SI) and the wildtype strain $(D K)$ in mixture (Figure S5B). Except for SI11 $(t$ test, $p=0.29)$, the $W_{S I-D K}$ values were all negative ( $t$-test, $p=0.04$ for SI08; $p<0.01$ for the other SI mutants). To eliminate the effects of differences in growth ability between partners, we calculated the $C_{S I}(D K)$ and $C_{D K}(S I)$ values to estimate the strains' competitive efficiencies in their mixtures (Figure 4A). Compared with their monocultures, SI01, SI02, SI07, SI08, and SI09 showed significantly decreased growth abilities when mixed with DK1622, while the SI03 and SI11 mutants significantly increased their growth $(t$-test, $p<0.01)$. When mixed with DK1622, the growth abilities of SI04 and SI06 were not significantly different from those in their monocultures ( $t$-test, $p=0.63$ for SI04, $p=0.19$ for SI06). However, in contrast to the sporulation increase, DK1622 significantly decreased its growth in each of the mixtures with the SI mutants ( $t$-test, $p=0.02$ for SI03, $p=0.03$ for SI09; $p<0.01$ for other SI mutants). The growth competition abilities between the SI mutants and the wild-type strain DK1622 in each of the mixtures, represented by the $C_{S I-D K}$ values, are shown in Figure 4B. Similarly, the $C_{S I-D K}$ and $W_{S I-D K}$ growth parameters were strongly correlated $(r=0.95, p<0.01)$. Four strains, SI01, SI02, SI07, and SI09, showed significantly weaker growth abilities than DK1622 $(t$ test, $p=0.02$ for SI02 and SI09; $p<0.01$ for other two), while the SI03, SI08 and SI11 strains grew significantly better than DK1622 in their co-cultures $(t$-test, $p<0.01)$. The competitive growth abilities of SI04 and SI06 in their mixtures with DK1622 were not significantly different from that of DK1622 ( $t$-test, $p=0.23$ for the SI04-DK1622 pair, $p=0.06$ for the SI06-DK1622 pair).

Although the $C_{S I-D K}$ values for growth were always larger than those for sporulation, the differences in competitive growth ability between the SI mutants and DK1622 were highly correlated with their sporulation efficiency differences $(r=0.89$, $p<0.01$; Figure 5), thus demonstrating the consistency of competitive abilities between the SI mutants and the wild-type strain under the growth and development conditions. Overall, we can simply divide the competitive abilities between the SI mutants and DK1622 into three types: the competitive efficiency of the mutant was lower than that of DK1622 (SI01, SI02, SI07, and SI09; $C_{S I-D K}<0$ ), higher than that of DK1622 (SI03 and SI11; $C_{S I-D K}>0$ ), and lower for sporulation but higher for growth than that of DK1622 (SI04, SI06, and SI08). Thus, compared with the DK1622 partner, the competitive abilities of the SI mutants were improved, reduced or unchanged in the mixtures. Notably, the competitive abilities of the mutants in the mixtures were not in line with their growth or sporulation abilities in the monocultures. For example, while SI09 and DK1622 yielded similar CFUs in their monocultures, the mutant had a weaker competitive growth ability than DK1622 in the mixture. Similarly, in their monocultures, SI07 and DK1622 had similar sporulation abilities, but the mutant had markedly repressed sporulation ability when mixed with DK1622 for codevelopment.

We also checked the survival of partners in pairwise mixtures of the nine independent SI mutants using strain-specific PCR amplifications (Figure 6 \& Figure S6). All of the SI mutants except SI01 and SI07 were detectable in mixtures with any other SI mutant after 48-h incubation on the CTT growth medium. When the SI01 mutant was mix-cultivated with other SI mutants, the SI01-specific PCR amplification produced virtually no product in the mixtures with other mutants except for the band in the SI01/SI07 mixture. In the mixtures of SI07 with other SI mutants, except the SI01/SI07 pair, the SI07-specific sequence was either weakly amplified (in the mixtures with SI03, SI04, SI06, and SI11) or undetectable (in other mixtures) after 48-h incubation on the CTT growth medium. In the SI01/SI07 mixture, the SI01-specific amplification band was weak, and the SI07-specific amplification band was as bright as that of the positive control. The above results clearly indicate that the mutations in SI01 and SI07 caused the mutants to be damaged and consequently exploited by the mixture partners. When SI01 and SI07 were co-cultured, the SI07 mutant survived. 

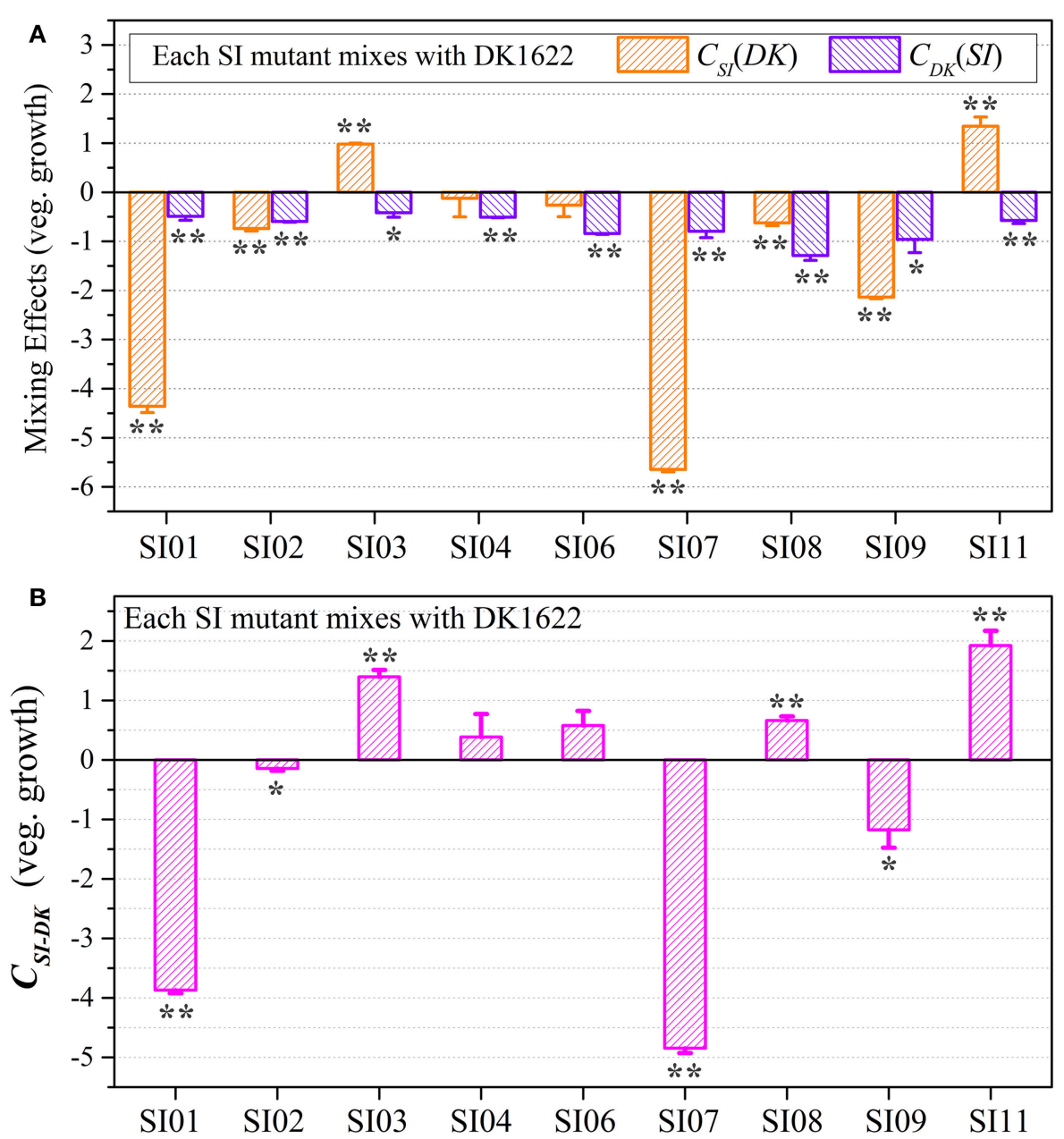

FIGURE 4 | Competitive growth abilities of incompatible mutants and DK1622 in paired mixtures. (A) The log-scale difference between each strain's growth ability in mixture and in monoculture. (B) The competitive growth differences of SI mutants and DK1622 in 1:1 mixtures under vegetative growth conditions after eliminating the strain's growth difference in monoculture. Three dilutions and three replications were performed for each assay, and the error bars represent the standard deviations. Asterisks denote the $p$-values for $t$-tests of differences from zero: ${ }^{\star} p<0.05,{ }^{* \star} p<0.01$.

\section{DISCUSSION}

To date, there have been few reports on bacterial colonymerger incompatibility (Gibbs et al., 2008; Gibbs and Greenberg, 2011; Rendueles et al., 2015; Lyons et al., 2016). Diverse incompatible strains within bacterial species in small soil niches (Vos and Velicer, 2009; Stefanic et al., 2015) suggest that sibling incompatibility probably results from various genetic mechanisms. Using laboratory evolution experiments with a strain of $M$. xanthus, Rendueles et al., showed that this bacterium can easily develop into colony-merger incompatible spontaneous mutants, probably involving diverse molecular genetic mechanisms (Rendueles et al., 2015). In this study, we obtained multiple independent incompatible strains from the mutations of $M$. xanthus DK1622. These incompatible strains formed obvious colony boundaries with the ancestral strain as well as with each other, whereas the boundary did not occur between two colonies of the same strain. The mutations were scattered throughout the genome and none of them has been genetically or biochemically studied previously. The SI mutants showed varied growth abilities and retained their multicellular swarming and development abilities in their monocultures. We calculated the competitive efficiencies of SI mutants mixed with DK1622 and found that most of the mutants showed improved or reduced competitive abilities compared with the ancestral strain, but some mutants were not affected by the DK1622 pairing under either the growth or the development conditions. For example, while the growth and sporulation abilities of SI01 and SI07 were largely repressed, the growth abilities of SI04 and SI06 and the sporulation abilities of SI09 were minimally affected when mixed with DK1622. Notably, the competitive abilities of the SI mutants in mixtures were inconsistent with their growth or development abilities in monocultures.

A number of killing strategies have been shown to be involved in competitive interactions between incompatible bacterial siblings. The first mechanism to have been intensively studied 


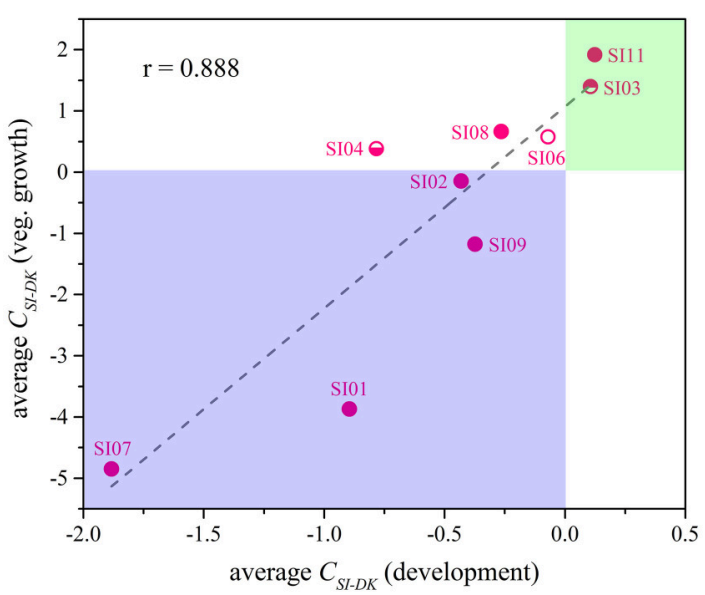

FIGURE 5 | The competitive abilities between the incompatible mutants and the wild-type strain under vegetative growth and development conditions. The average $\mathrm{C}_{S I-D K}$ values during vegetative growth (y-Axis) strongly correlated with the values during co-development (x-Axis; $r=0.89, p<0.01)$.

is the production of small antimicrobial compounds, which act as antimicrobials or signal molecules under different conditions (Hibbing et al., 2010; Cornforth and Foster, 2013). In addition to the production of small molecule antibiotics, space competition, predation and direct poison delivery have also been reported to participate in cannibalistic interactions, such as contactdependent inhibition and the type VI secretion system (Hayes et al., 2010; Konovalova and Sogaard-Andersen, 2011; Basler et al., 2013; Ho et al., 2014; Russell et al., 2014; Willett et al., 2015). Kin cells could be protected from the toxic proteins delivered by these pathways using specific cognate immunity proteins (Hood et al., 2010; Russell et al., 2011). Based on the survival of partners in mixture, we suggest that the systems involved in the colony-merger incompatibility of $M$. xanthus strains might include not only some kind of killing pathway for the strongly repressive effects observed in SI01 and SI07, but also some unknown mechanisms with almost no repression, as in the case of SI11. Other mechanisms that underlie the moderate interactions between other pairs could also be involved in the colony-merger incompatibilities. Some mutated genes are annotated to encode putative enzymes; for example, the mutated MXAN_0390 gene in SI03 is annotated to encode a putative regulator of ribonuclease A, and the MXAN_7251 gene in SI11 is annotated to encode a serine/threonine protein kinase. Some regulator genes have been shown to be involved in the kin discrimination between siblings in B. subtilis (Lyons et al., 2016). Additional studies are needed to identify the molecular mechanisms of kin discrimination in M. xanthus DK1622.

Individual organisms compete with their neighbors for shared space and food. Mixing the incompatible strains together may reduce the survival chances of one or both or even lead to the death of one or the other (Vos and Velicer, 2009; Li et al., 2013). However, incompatible $M$. xanthus strains are unable to occupy identical niches (Vos and Velicer, 2009). The competitive interactions between incompatible strains derived from $M$. xanthus DK1622 could provide a suggestion for the ecological co-existence strategy of naturally incompatible strains. The results of the current study provide evidence that the relationships between incompatible $M$. xanthus strains are diverse. Some DK1622-derived SI mutants might not survive in mixtures with DK1622, which suggests that long-term co-growth might lead to the disappearance of the inferior strains. However, separate territories, as well as the boundaries between colonies, are able to prevent the vulnerable incompatible strains from being digested by the surrounding predatory $M$. xanthus cells. We assume that after adaptive evolution in natural environments, incompatible Myxococcus strains localize and establish separate niche territories. The colony boundaries provide physical barriers to hinder colony mixing and avoid cannibalism, and thus allow more opportunities for separate kin groups to expand the genetic diversity within the population (Kraemer et al., 2016; Velicer and Plucain, 2016; Wielgoss et al., 2016). When the environment changes, such genetic diversity will increase the adaptation of the whole population.

In conclusion, in this study we demonstrated that multiple colony-merger incompatibilities in $M$. xanthus are associated with different genetic loci. Diverse competitive interactions between the incompatible $M$. xanthus strains indicated that the loss of compatibility might have a broad range of effects on the fitness of the mutants. The results of the incompatible $M$. xanthus mutants derived from DK1622 suggest that the relationships and ecological co-existence strategies of incompatible bacterial strains are diverse in the natural environment.

\section{MATERIALS AND METHODS}

\section{Bacterial Strains and Culture Conditions}

The bacterial strains and plasmids used in this work are listed in Table S1. M. xanthus DK1622 and its mutants were cultivated at $30^{\circ} \mathrm{C}$ in liquid CTT medium (Hodgkin and Kaiser, 1977). Hard CTT medium was prepared with an agar concentration of $1.5 \%$, while the soft CTT plate contained $0.3 \%$ agar. E. coli strains were cultivated in lysogeny broth (LB) medium or on solid LB medium. If required, CTT and LB media were supplemented with kanamycin $\left(\mathrm{Km} ; 40 \mu \mathrm{g} \mathrm{ml}^{-1}\right)$.

\section{Screening for Self-Identification Mutants}

The mutants were generated using the random transposon plasmid, pMiniHimar-lacZ (Chavira et al., 2007). Transformants were inoculated next to each other on CTT agar plates supplemented with $\mathrm{Km}$ to screen for mutants in colony-merger incompatibility conditions. Colonies of mutants that could not merge with their neighbors were inoculated into liquid CTT medium containing $\mathrm{Km}$ and shaken at $200 \mathrm{rpm}$ for $24 \mathrm{~h}$. The dispersed cells were sampled, centrifuged and suspended in TPM buffer at a final concentration of $5 \times 10^{9}$ cells $/ \mathrm{ml}$. Three microliter aliquots of cell suspension were pairwise inoculated on CTT plates adjacent to another cell suspension, separated by a distance of $7 \mathrm{~mm}$. After 5 days of cultivation at $30^{\circ} \mathrm{C}$, boundaries were observed under a SMZ100 dissection microscope. The mutants that were incompatible with their neighbors were selected for further neighboring inoculation with the wild-type 


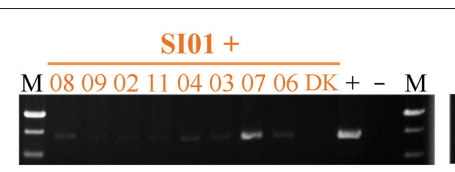

$\mathrm{SI04}+$

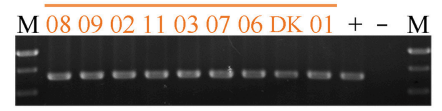

SI08+

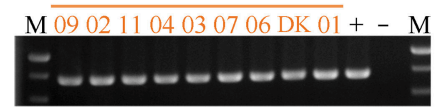

$\mathrm{SI} 02+$

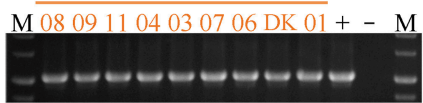

SI06 +

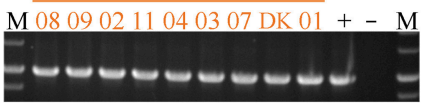

SI09+

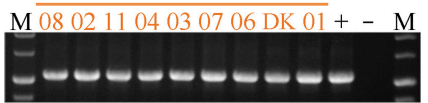

$\mathrm{SI} 03+$

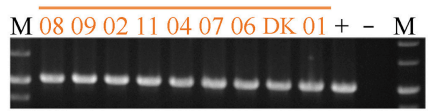

$\mathrm{SI07+}$

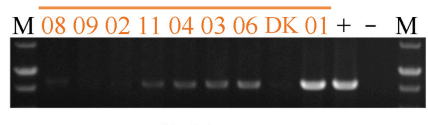

$\mathrm{SI} 11+$

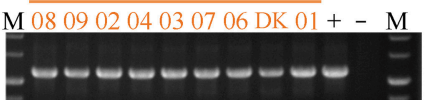

FIGURE 6 | Strain-specific PCR amplification during vegetative growth to assay the presence of partners in mixed cultures among incompatible mutants. The number represents the specific SI mutant; i.e., 01 corresponds to SI01, 02 to SI02, etc. The + symbol indicates that the strain was mixed with an equal volume of TPM buffer as the positive control. The - symbol indicates the blank DK1622 control genome. M, molecular weight markers.

strain DK1622, and the mutants that formed visually apparent boundaries with wild-type DK1622 were stored for further analyses.

\section{Dyeing Assay of Colony Boundaries}

The fate of boundary cells was determined using LIVE/DEAD ${ }^{\circledR}$ BacLightTM Bacterial Viability Kits, L7012 (Invitrogen, USA) following the manufacturer's fluorescence microscopy protocol with some modifications. SYTO 9 and propidium iodide dyes were mixed in equal volumes. Three microliters of the mixture were diluted in $1 \mathrm{ml}$ TPM buffer and dropped onto a cover slip. The agar between two strain colonies where the boundary was formed was excised and placed upside-down on a drop of dye mixture in the dark for $15 \mathrm{~min}$, and then observed under a fluorescence inverse microscope equipped with a B-2A standard filter (Nikon, Japan).

\section{Southern Blot}

To identify the single insertion site, the genomic DNAs of the strains were isolated, digested by SacII, separated in a $1 \%$ agarose gel and transferred to a Hybond- $\mathrm{N}^{+}$transfer membrane (Amersham Biosciences, UK). The membrane was probed with a digoxigenin (DIG)-labeled primer amplified from the alph gene in pMiniHimar-lacZ. Immunological detection was performed according to the manual provided with the DIG High Prime DNA Labeling and Detection Starter Kit I (Roche, USA).

\section{Mapping the Locations of the Insertion Mutants}

The insertion sites of the colony-merger incompatible mutants were localized by plasmid rescuing and sequencing (Pan et al., 2010). Briefly, the genomic DNA of mutants was extracted using the cetyltrimethyl ammonium bromide method and digested with SacII. After purification by alcohol precipitation, fragments were self-circularized using T4 DNA ligase. The ligation product was transformed into the $E$. coli $\mathrm{DH} 5 \alpha \lambda$ pir strain. After cultivation on LB plates containing $\mathrm{Km}$ for $\sim 14$ h, five colonies were selected randomly for the extraction of plasmid DNA using the eZNA Plasmid Mini Kit I (Omega Bio-Tek) according to the manufacturer's instructions, and then sequenced using the primer 5'-GAA CTA TGT TGA ATA ATA AAA ACG-3'.

\section{Phenotypic Characteristics of Mutants}

The mutants and the wild-type strains were assessed for motility phenotypes using standard methods (Shi and Zusman, 1993). To assay the cell-swarming capacity, aliquots $\left(2 \mu \mathrm{l}, 5 \times 10^{9}\right.$ cells $\left./ \mathrm{ml}\right)$ of cells were inoculated onto CTT medium containing 1.5 or $0.3 \%$ agar. After 5 days of incubation at $30^{\circ} \mathrm{C}$, we measured the sizes of swarming colonies using a Nikon D60 camera (Nikon, Japan).

Developmental ability was assessed on TPM plates using the previously described methods (Kroos et al., 1986). The strains were inoculated on CTT or CTT $+\mathrm{Km}$ plates for 3 days and then transferred into liquid CTT or CTT $+\mathrm{Km}$ medium. Cultures were centrifuged and resuspended in TPM buffer to a density of $\sim 5 \times 10^{9}$ cells $/ \mathrm{ml}$. Five microliters of the cell suspension were inoculated onto a TPM plate and incubated for 5 days. Five dots from each plate were harvested and resuspended in $100 \mu \mathrm{l}$ of TPM buffer and then lightly sonicated to a homogenized state. After incubation for $2 \mathrm{~h}$ at $50^{\circ} \mathrm{C}$, the cells were serially diluted, mixed with CTT containing $0.3 \%$ agar and poured into CTT and CTT $+\mathrm{Km}$ plates. Counting of the colonies was performed. Three dilutions and three replications were used.

\section{Pilus Formation Ability}

The pilus from the strains were purified using the surface pilus preparation method described previously (Chavira et al., 2007). Pilus from DK1622 and SW504 ( $\triangle$ difA) were prepared as positive controls, and pilus from DK10410 ( $\Delta$ pilA) was prepared as a negative control. Western blotting was prepared using standard procedures with a $1 / 2,000$ dilution of anti-PilA serum (Jafari et al., 2014).

\section{EPS Production Detection}

For the EPS analysis, cells collected from CTT cultures were washed with TPM buffer and adjusted to a density of $5 \times 10^{9}$ cells/ml. A $10 \mu \mathrm{l}$ aliquot of cell suspension was spotted onto CTT plates containing $50 \mu \mathrm{g} \mathrm{ml}^{-1}$ of Calcofluor white M2R. After 
incubation at $30^{\circ} \mathrm{C}$ for 5 days, the plate cultures were detected under $365 \mathrm{~nm}$ ultraviolet light. To quantitatively analyze the EPS, a previously described method (Black and Yang, 2004) was used to bind Congo red dye to the EPS (Dana and Shimkets, 1993; Yang et al., 2000).

\section{Construction of $M$. xanthus Mutants}

Deletion mutations were generated in the wild-type strain DK1622 using standard methods (Wu and Kaiser, 1996). Upstream and downstream regions of genes were amplified, ligated together, and cloned into the plasmid pBJ113. The deletion plasmid was electroporated into DK1622, where it was integrated into the genome by homologous recombination. The strains growing on CTT plates containing $\mathrm{Km}$ were picked and then screened on 1\% D-galactose CTT agar plates. The strains growing on CTT plates were then picked and checked by the colony PCR.

\section{Mixing Experiments for Sporulation}

Strains were inoculated into the liquid CTT medium, and shaken at $30^{\circ} \mathrm{C}$ for $24 \mathrm{~h}$ to the mid-log growth phase. After being harvested by centrifugation at $8,000 \times \mathrm{g}$ for $5 \mathrm{~min}$, the cells were resuspended in the TPM buffer to a high density of $5 \times 10^{9}$ cells $/ \mathrm{ml}$. The cell suspensions of pairing strains were mixed at the ratio of 1:1 (v/v), and 10-microliter aliquots of each mixture were inoculated onto TPM plates, which were incubated for 5 days for sporulation.

To calculate the number of developed myxospores, five dots of each mixed culture were harvested and suspended in $100 \mu \mathrm{l}$ of the TPM buffer. The suspensions were blown by the pipettor, mixed completely in a vortex mixer for $10 \mathrm{~s}$, and then dispersed with a sonicator. The cell suspensions were heated at $50^{\circ} \mathrm{C}$ for $2 \mathrm{~h}$ to kill the vegetative cells. After 10 -fold serial dilution, 50 microliters of the cell suspension were mixed with $2.5 \mathrm{ml}$ of molten CTT soft agar and the mixtures were immediately poured onto CTT hard agar plates with $\mathrm{Km}$ (the growing cells were the insertion mutants) and CTT plates without the antibiotic (the growing cells included the insertion mutants and the wild-type strain DK1622). After 5 days of incubation at $30^{\circ} \mathrm{C}$, the CFUs were counted to determine the sporulation abilities of strains. Three dilutions of the mixed spore suspensions and three replications for each dilution were used for the counting.

\section{Mixing Experiments for Vegetative Growth}

Cells were shaken in liquid CTT growth medium at $30^{\circ} \mathrm{C}$ for $24 \mathrm{~h}$ to the mid-log phase. After centrifugation, the harvested cells were resuspended in the TPM buffer and adjusted to a density of $5 \times 10^{9}$ cells $/ \mathrm{ml}$. The cell suspensions of pairing strains were mixed at a $1: 1(\mathrm{v} / \mathrm{v})$ ratio. Five-microliter aliquots of the mixed suspensions were dropped onto CTT agar medium. After $48 \mathrm{~h}$ of incubation at $30^{\circ} \mathrm{C}$, the entire colonies were harvested, suspended in $500 \mu 1$ TPM buffer and then 10 -fold serially diluted in TPM buffer. To calculate the survival partner cells, cell diluents $(50 \mu \mathrm{l})$ were mixed with $2.5 \mathrm{ml}$ molten CTT soft agar and poured onto CTT hard agar plates with $\mathrm{Km}$ or with no added antibiotic. After 5 days of incubation at $30^{\circ} \mathrm{C}$, the $\mathrm{CFUs}$ of the vegetative growth were counted. Three dilutions and three replications for each dilution were used for the counting.

\section{Competitive Ability Conduction}

We measured the competition between different strains using the previously described method (Fiegna and Velicer, 2005) with some modifications. $N_{S I}$ and $N_{D K}$ represent the cell number of an SI mutant or DK1622 before pairwise mixing of the culture $\left(t_{0}\right)$ and the CFUs after $48 \mathrm{~h}$ of cultivation $\left(t_{2}\right)$ for vegetative growth or 5 days of culture $\left(t_{5}\right)$ for sporulation. The growth or sporulation ability $(D)$ of an SI mutant or DK1622 in the monoculture is given as

$$
\begin{aligned}
D_{S I} & =N_{S I}\left(t_{n}\right) / N_{S I}\left(t_{0}\right) \\
D_{D K} & =N_{D K}\left(t_{n}\right) / N_{D K}\left(t_{0}\right)
\end{aligned}
$$

The growth or sporulation ability of an SI mutant or DK1622 in the co-culture is similarly given as

$$
\begin{aligned}
& D_{S I}(D K)=N_{S I}\left(D K, t_{n}\right) / N_{S I}\left(D K, t_{0}\right) \\
& D_{D K}(S I)=N_{D K}\left(S I, t_{n}\right) / N_{D K}\left(S I, t_{0}\right)
\end{aligned}
$$

The difference in the growth or sporulation competitive ability of an SI mutant and DK1622 in the co-culture is defined as

$$
W_{S I-D K}=\log \left(D_{S I}[D K]\right)-\log \left(D_{D K}[S I]\right)
$$

The growth or sporulation efficiency of an SI mutant and DK1622 in the mixture after eliminating the growth or sporulation difference in the monoculture is given as

$$
\begin{aligned}
& C_{S I}(D K)=\log \left(D_{S I}[D K]\right)-\log \left(D_{S I}\right) \\
& C_{D K}(S I)=\log \left(D_{D K}[S I]\right)-\log \left(D_{D K}\right)
\end{aligned}
$$

Thus, a positive value of $C_{S I}(D K)$ indicates that the SI mutant grows or sporulates more efficiently in the mixture with DK1622 than in the monoculture, whereas a negative value indicates that mixing with DK1622 negatively affects the efficiency of the SI mutant. $C_{D K}(S I)$ has a similar meaning. After eliminating the growth or sporulation difference of each strain in the monoculture, the competitive ability between the SI mutants and the wild-type strain DK1622 in the co-culture is given as

$$
C_{S I-D K}=C_{S I}(D K)-C_{D K}(S I)
$$

\section{Competitive Growth Ability Assays by Strain-Specific PCR}

To perform the strain-specific PCR amplifications to determine the survival of partners in pairwise mixtures co-cultivated for 2 days between the nine independent SI mutants, we designed the specific primers listed in Table S2. The upstream primer was the sequence from the random transposon plasmid, pMiniHimarlacZ, and was the same for all of the mutants. However, the downstream primers were specific for each mutant and were the sequence from the inserted genes. 


\section{Statistical Analysis}

To examine the significance of differences, Student's $t$-tests were conducted using SPSS software. Differences were considered significant and highly significant at $p<0.05$ and $<0.01$, respectively.

\section{AUTHOR CONTRIBUTIONS}

YG and YL designed experiments. YG, XZ, MA, XH, ZL, and XC performed experiments. ZZ, YG, XZ, and YL analyzed data. YL, $\mathrm{YG}$, and $\mathrm{ZZ}$ wrote the paper.

\section{ACKNOWLEDGMENTS}

This work was financially supported by the National Natural Science Foundation of China (NSFC) (No. 31471183 and 31670076), the NSFC Key Program (No. 31130004) and the Key Program of Shandong Natural Science Foundation (No. ZR2016QZ002) awarded to YL.

\section{SUPPLEMENTARY MATERIAL}

The Supplementary Material for this article can be found online at: https://www.frontiersin.org/articles/10.3389/fmicb. 2018.01200/full\#supplementary-material

Video S1 | Boundary formation between SI01 (left) and DK1622 (right).

Figure S1 | A clear red line appeared in the colony boundary between DK1622 and SI04. The microscopic images were taken after dyeing with a mixture of SYTO 9 for live cells (in green) and propidium iodide for damaged cells or cells with an incomplete membrane (in red). The lower panels (60 × magnification) are higher

\section{REFERENCES}

Basler, M., Ho, B. T., and Mekalanos, J. J. (2013). Tit-for-tat: type VI secretion system counterattack during bacterial cell-cell interactions. Cell 152, 884-894. doi: 10.1016/j.cell.2013.01.042

Black, W. P., and Yang, Z. (2004). Myxococcus xanthus chemotaxis homologs DifD and DifG negatively regulate fibril polysaccharide production. J. Bacteriol. 186, 1001-1008. doi: 10.1128/JB.186.4.1001-1008.2004

Brown, S. P., and Buckling, A. (2008). A social life for discerning microbes. Cell 135, 600-603. doi: 10.1016/j.cell.2008.10.030

Chavira, M., Cao, N., Le, K., Riar, T., Moradshahi, N., McBride, M., Lux, R., et al. (2007). Beta-D-Allose inhibits fruiting body formation and sporulation in Myxococcus xanthus. J. Bacteriol. 189, 169-178. doi: 10.1128/JB.00 792-06

Cornforth, D. M., and Foster, K. R. (2013). Competition sensing: the social side of bacterial stress responses. Nat. Rev. Microbiol. 11, 285-293. doi: $10.1038 / \mathrm{nrmicro} 2977$

Dana, J. R., and Shimkets, L. J. (1993). Regulation of cohesion-dependent cell interactions in Myxococcus xanthus. J. Bacteriol. 175, 3636-3647. doi: $10.1128 /$ jb.175.11.3636-3647.1993

Dienes, L. (1946). Reproductive processes in Proteus cultures. Proc. Soc. Exp. Biol. Med. 63, 265-270. doi: 10.3181/00379727-63-15570

Dworkin, M. (1996). Recent advances in the social and developmental biology of the myxobacteria. Microbiol. Rev. 60, 70-102.

Fiegna, F., and Velicer, G. J. (2005). Exploitative and hierarchical antagonism in a cooperative bacterium. PLoS Biol. 3:e370. doi: 10.1371/journal.pbio.0030370

Gibbs, K. A., and Greenberg, E. P. (2011). Territoriality in Proteus: advertisement and aggression. Chem. Rev. 111, 188-194. doi: 10.1021/cr100051v magnifications of the upper image $(10 \times$ magnification). Scale bars, 0.5 and $0.1 \mathrm{~mm}$.

Figure S2 | The boundary formation phenotypes of the deletion mutations of the six mutated genes inserted in M. xanthus DK1622: the MXAN_0049 gene in the SI01 mutant, MXAN_0085 in SI02, MXAN_RS36575 in SI05, MXAN_2099 in SI07, MXAN_RS24590 in SI08 and MXAN RS34540 in SI10.

Figure S3 | The production of PilA proteins and EPS. (A) Western blotting determined the presence of extracellular type IV pili (TFP) in SI mutants, broken down from vortexed cells. SW504 ( $\Delta$ difA), DK10410 ( $\Delta$ pilA), and the wild-type strain DK1622 were used as controls. The yields of extracellular polysaccharides (EPS) in SI mutants were detected using Congo red binding (B) and calcium fluorescence staining (C).

Figure S4 | Sporulation abilities of mixed incompatible mutants and their ancestral strain DK1622 on the TPM medium. (A) The relative sporulation values of each partner in the co-development. (B) Differences in the sporulation abilities of co-cultured partners. Three dilutions and three replications were performed. Error bars represent standard deviations, and asterisks denote $p$-values for $t$-tests of differences from zero: ${ }^{* *} p<0.01$.

Figure S5 | Growth abilities of incompatible mutants and DK1622 in paired mixtures. (A) CFU numbers of paired cultured strains. SI mutants and DK1622 were mixed at ratios of 1:1 (V/v). (B) Differences in the growth abilities of incompatible mutants $(S /)$ and DK1622 (DK) in 1:1 mixtures during vegetative growth. Three dilutions and three replications were performed for each assay. Error bars represent standard deviations. Asterisks denote $p$-values for $t$-tests of differences from zero: ${ }^{*} p<0.05,{ }^{* *} p<0.01$.

Figure S6 | The original gel images of the strain-specific PCR amplification products, which were analyzed by agarose gel electrophoresis and ethidium bromide staining. The number represents the specific SI mutant; i.e., 01 corresponds to SI01, 02 to SIO2, etc. The + and - symbols indicate the positive and negative controls, respectively. M: molecular weight markers.

Table S1 | Strains and plasmids used in this study.

Table S2 | Primers used in this study.

Gibbs, K. A., Urbanowski, M. L., and Greenberg, E. P. (2008). Genetic determinants of self identity and social recognition in bacteria. Science 321, 256-259. doi: 10.1126/science.1160033

Goldman, B. S., Nierman, W. C., Kaiser, D., Slater, S. C., Scott Durkin, A., Eisen, J. A., et al. (2006). Evolution of sensory complexity recorded in a myxobacterial genome. Proc. Natl. Acad. Sci. U.S.A. 103, 15200-15205. doi: 10.1073/pnas.0607335103

Griffin, A. S., West, S. A., and Buckling, A. (2004). Cooperation and competition in pathogenic bacteria. Nature 430, 1024-1027. doi: 10.1038/nature02744

Hamilton, W. D. (1964). The genetical evolution of social behaviour. I. J. Theor Biol. 7, 1-16. doi: 10.1016/0022-5193(64)90038-4

Hayes, C. S., Aoki, S. K., and Low, D. A. (2010). Bacterial contactdependent delivery systems. Annu. Rev. Genet. 44, 71-90. doi: 10.1146/annurev.genet.42.110807.091449

Hibbing, M. E., Fuqua, C., Parsek, M. R., and Peterson, S. B. (2010). Bacteria competition: surviving and thriving in the microbial jungle. Nat. Rev. Microbiol. 8, 15-25. doi: $10.1038 /$ nrmicro2259

Ho, B. T., Dong, T. G., and Mekalanos, J. J. (2014). A view to a kill: the bacterial type VI secretion system. Cell Host Microbe 15, 9-21. doi: 10.1016/j.chom.2013.11.008

Hodgkin, J., and Kaiser, D. (1977). Cell-to-cell stimulation of movement in nonmotile mutants of Myxococcus. Proc. Natl. Acad. Sci. U.S.A. 74, 2938-2942. doi: 10.1073/pnas.74.7.2938

Hood, R. D., Singh, P., Hsu, F., Güvener, T., Carl, M. A., Trinidad, R. R., et al (2010). A type VI secretion system of Pseudomonas aeruginosa targets a toxin to bacteria. Cell Host Microbe 7, 25-37. doi: 10.1016/j.chom.2009.12.007

Jafari, R., Almqvist, H., Axelsson, H., Ignatushchenko, M., Lundbäck, Nordlund, P., and Martinez Molina, D. (2014). The cellular thermal shift assay for 
evaluating drug target interactions in cells. Nat. Protocols 9, 2100-2122. doi: $10.1038 /$ nprot.2014.138

Keller, L., and Surette, M. G. (2006). Communication in bacteria: an ecological and evolutionary perspective. Nat. Rev. Microbiol. 4, 249-258. doi: 10.1038/nrmicro1383

Konovalova, A., and Sogaard-Andersen, L. (2011). Close encounters: contact-dependent interactions in bacteria. Mol. Microbiol. 81, 297-301. doi: 10.1111/j.1365-2958.2011.07711.x

Kraemer, S. A., Wielgoss, S., Fiegna, F., and Velicer, G. J. (2016). The biogeography of kin discrimination across microbial neighbourhoods. Mol. Ecol. 25, 4875-4888. doi: 10.1111/mec.13803

Kroos, L., Kuspa, A., and Kaiser, D. (1986). A global analysis of developmentally regulated genes in Myxococcus xanthus. Dev. Biol. 117, 252-266. doi: 10.1016/0012-1606(86)90368-4

Li, P. F., Li, S. G., Li, Z. F., Zhao, L., Wang, T., Pan, H. W., et al. (2013). Co-cultivation of Sorangium cellulosum strains affects cellular growth and biosynthesis of secondary metabolite epothilones. FEMS Microbiol. Ecol. 85, 358-368. doi: 10.1111/1574-6941.12125

Li, Y., Sun, H., Ma, X., Lu, A., Lux, R., Zusman, D., et al. (2003). Extracellular polysaccharides mediate pilus retraction during social motility of Myxococcus xanthus. Proc. Natl. Acad. Sci. U.S.A. 100, 5443-5448. doi: 10.1073/pnas.0836639100

Lyons, N. A., Kraigher, B., Stefanic, P., Mandic-Mulec, I., and Kolter, R. (2016). A combinatorial kin discrimination system in Bacillus subtilis. Curr. Biol. 26, 733-742. doi: 10.1016/j.cub.2016.01.032

Munson, E. L., Pfaller, M. A., and Doern, G. V. (2002). Modification of dienes mutual inhibition test for epidemiological characterization of Pseudomonas aeruginosa isolates. J. Clin. Microbiol. 40, 4285-4288. doi: 10.1128/JCM.40.11.4285-4288.2002

Pan, H. W., Tan, Z. G., Liu, H., Li, Z. F., Zhang, C. Y., Li, C. Y., et al. (2010). Hdsp, a horizontally transferred gene required for social behavior and halotolerance in salt-tolerant Myxococcus fulvus HW-1. ISME J. 4, 1282-1289. doi: 10.1038/ismej.2010.52

Rendueles, O., Zee, P. C., Dinkelacker, I., Amherd, M., Wielgoss, S., and Velicer, G. J. (2015). Rapid and widespread de novo evolution of kin discrimination. Proc. Natl. Acad. Sci. U.S.A. 112, 9076-9081. doi: 10.1073/pnas.1502251112

Rubin, E. J., Akerley, B. J., Novik, V. N., Lampe, D. J., Husson, R. N., and Mekalanos, J. J. (1999). In vivo transposition of mariner-based elements in enteric bacteria and mycobacteria. Proc. Natl. Acad. Sci. U.S.A .96, 1645-1650. doi: 10.1073/pnas.96.4.1645

Russell, A. B., Hood, R. D., Bui, N. K., LeRoux, M., Vollmer, W., and Mougous, J. D. (2011). Type VI secretion delivers bacteriolytic effectors to target cells. Nature 475, 343-347. doi: 10.1038/nature10244

Russell, A. B., Peterson, S. B., and Mougous, J. D. (2014). Type VI secretion system effectors: poisons with a purpose. Nat. Rev. Microbiol. 12, 137-148. doi: $10.1038 /$ nrmicro3185

Shi, W., and Zusman, D. R. (1993). The two motility systems of Myxococcus xanthus show different selective advantages on various surfaces. Proc. Natl. Acad. Sci. U.S.A. 90, 3378-3382. doi: 10.1073/pnas.90.8.3378

Shimkets, L. J. (1990). Social and developmental biology of the myxobacteria. Microbiol. Rev. 54, 473-501.

Smith, D. R., and Dworkin, M. (1994). Territorial interactions between two Myxococcus Species. J. Bacteriol. 176, 1201-1205. doi: 10.1128/jb.176.4.1201-1205.1994

Stefanic, P., Kraigher, B., Lyons, N. A., Kolter, R., and Mandic-Mulec, I. (2015). Kin discrimination between sympatric Bacillus subtilis isolates. Proc. Natl. Acad. Sci. U.S.A. 112, 14042-14047. doi: 10.1073/pnas.1512671112
Strassmann, J. E., Gilbert, O. M., and Queller, D. C. (2011). Kin discrimination and cooperation in microbes. Annu. Rev. Microbiol. 65. 349-367. doi: 10.1146/annurev.micro.112408.134109

Velicer, G. J., and Plucain, J. (2016). Evolution: bacterial territoriality as a byproduct of kin discriminatory warfare. Curr. Biol. 26, R364-R366. doi: 10.1016/j.cub.2016.03.033

Velicer, G. J., and Vos, M. (2009). Sociobiology of the myxobacteria. Annu. Rev. Microbiol. 63, 599-623. doi: 10.1146/annurev.micro.091208.0 73158

Vos, M., and Velicer, G. J. (2009). Social conflict in centimeter-and global-scale populations of the bacterium Myxococcus xanthus. Curr. Biol. 19, 1763-1767. doi: $10.1016 /$ j.cub.2009.08.061

Waibel, M., Floreano, D., and Keller, L. (2011). A quantitative test of Hamilton's rule for the evolution of altruism. PLoS Biol. 9:e1000615. doi: 10.1371/journal.pbio.1000615

West, S. A., Diggle, S. P., Buckling, A., Gardner, A., and Griffin, A. S. (2007). The social lives of microbes. Annu. Rev. Ecol. Evol. 38, 53-77. doi: 10.1146/annurev.ecolsys.38.091206.0 95740

West, S. A., Griffin, A. S., Gardner, A., and Diggle, S. P. (2006). Social evolution theory for microorganisms. Nat. Rev. Microbiol. 4, 597-607. doi: $10.1038 /$ nrmicro1461

Wielgoss, S., Didelot, X., Chaudhuri, R. R., Liu, X., Weedall, G. D., Velicer, G. J., et al. (2016). A barrier to homologous recombination between sympatric strains of the cooperative soil bacterium Myxococcus xanthus. ISME J. 10, 2468-2477. doi: 10.1038/ismej.2016.34

Willett, J. L., Gucinski, G. C., Fatherree, J. P., Low, D. A., and Hayes, C. S. (2015). Contact-dependent growth inhibition toxins exploit multiple independent cell-entry pathways. Proc. Natl. Acad. Sci. U.S.A. 112, 11341-11346. doi: $10.1073 /$ pnas. 1512124112

$\mathrm{Wu}$, S. S., and Kaiser, D. (1996). Markerless deletions of pil genes in Myxococcus xanthus generated by counterselection with the Bacillus subtilis sacB gene. J. Bacteriol. 178, 5817-5821. doi: 10.1128/jb.178.19.5817-582 1.1996

Wu, S. S., and Kaiser, D. (1997). Regulation of expression of the pilA gene in Myxococcus xanthus. J. Bacteriol. 179, 7748-7758. doi: 10.1128/jb.179.24.7748-7758.1997

Yang, Z., Ma, X., Tong, L., Kaplan, H. B., Shimkets, L. J., and Shi, W. (2000). Myxococcus xanthus dif genes are required for biogenesis of cell surface fibrils essential for social gliding motility. J. Bacteriol. 182, 5793-5798. doi: 10.1128/JB.182.20.5793-5798.2000

Youderian, P., Burke, N., White, D. J., and Hartzell, P. L. (2003). Identification of genes required for adventurous gliding motility in Myxococcus xanthus with the transposable element mariner. Mol. Microbiol. 49, 555-570. doi: 10.1046/j.1365-2958.2003.03582.x

Conflict of Interest Statement: The authors declare that the research was conducted in the absence of any commercial or financial relationships that could be construed as a potential conflict of interest.

Copyright (C) 2018 Gong, Zhang, Zhou, Anwar, Hu, Li, Chen and Li. This is an open-access article distributed under the terms of the Creative Commons Attribution License (CC BY). The use, distribution or reproduction in other forums is permitted, provided the original author(s) and the copyright owner are credited and that the original publication in this journal is cited, in accordance with accepted academic practice. No use, distribution or reproduction is permitted which does not comply with these terms. 